\title{
Mirror-reading and writing in association with right-left spatial disorientation
}

\author{
KENNETH M HEILMAN, GREGORY HOWELL, EDWARD VALENSTEIN, AND \\ LESLIE ROTHI
}

From the Department of Neurology, College of Medicine, University of Florida, and the Veterans Administration Medical Center, Gainesville, Florida, USA

SUMMARY A left-handed patient suddenly developed a right hemiparesis, mirror-reading, and mirror-writing. Although he could discriminate between right and left on himself, he demonstrated right/left spatial disorientation. We propose that his right/left spatial disorientation was induced by a scanning defect. It has been demonstrated that mirror-image engrams are normally available. We believe that a reversal of the learned left-to-right scanning process with the availability of mirror engrams induced mirror-reading. Similarly, reversal of the normal left-to-right writing pattern, with the availability of mirror engrams, induced mirror-writing.

"Mirror-writing" is script that runs in the direction opposite to normal, with the individual letters also reversed. It becomes legible to the normal reader when it is held to a mirror. "Mirror-reading" is when mirror-writing can be easily read without the aid of a mirror or when normally written English words are read right to left (for example "was" is read as "saw"). ${ }^{1}$

In 1698 Rosinus Lentilius described mirrorwriting 1 in a left-handed epileptic girl, and it has since been reported to be associated with various conditions. Mirror-writing can be accomplished by normal subjects by having them write on their forehead or under a table, or by practice. ${ }^{1}$ Mirror-writing may be seen in 2 to $3 \%$ of hemiplegic patients. ${ }^{2}{ }^{3}$ Retarded $^{4}$ or dyslexic ${ }^{5}$ children may read and write in this fashion, as may even normal children when they are learning to read and write. ${ }^{1}$

Although mirror-reading and writing are more common in left-handers, the mechanisms underlying these disorders have not been elucidated. ${ }^{6}$ Recently we observed a patient with the sudden onset of mirror-reading and writing. Analysis of this case has helped us gain further insight into the mechanism underlying these disorders.

Address for reprint requests : Dr KM Heilman, Department of Neurology, Box J236, J. Hillis Miller Health Center, University of Florida, Gainesville, FL 32610, USA.

Accepted 23 April 1980

\section{Case report}

The patient, a 58-year-old, left-handed man, had had four years of elementary education. There was no family history of left-handedness. Two days before admission he and his family noticed that he suddenly developed disordered speech, right-sided weakness, and difficulty in walking. During the next two days the patient's speech improved.

The past history was relevant in that at age 19 years, the patient had encephalitis and developed a tremor in both arms and the right leg. A left stereotaxic thalamotomy and pallidectomy was performed when he was age 39 , but it did not improve the tremor. The tremor was being treated with a combination of carbidopa and levodopa before the present episode, and except for the tremor and rigidity, his mental status and sensory and motor findings otherwise were normal.

General physical examination revealed a blood pressure of $160 / 105 \mathrm{~mm} \mathrm{Hg}$, but was otherwise normal. Neurological examination showed that the patient was lethargic. He was oriented for time, place and person, but had a poor fund of information (for example, he thought that George Wallace was president and the president before him was Abraham Lincoln). $\mathrm{He}$ had a digit span of six forward but was unable to recall any of three objects after being distracted 
for five minutes. Although speech was slightly dysarthric, he was not aphasic. When the patient signed the hospital admission form, he wrote his name in mirror-image fashion. This signature was compared with his previous signatures, which were written in the normal manner. $\mathrm{He}$ also wrote a spontaneous sentence in mirrorwriting. He wrote mirror-fashion with his left hand, the one with which he was accustomed to writing. He was able to read simple words, and given simple right-left commands, he appeared to know his right from left. Examination of the cranial nerves revealed the patient had an exotropia, decreased sensation on the right side of his face, and a slight asymmetry of his palate, the right side being lower than the left. There was a right hemiparesis, which was mild and appeared to maximally affect the distal upper extremity and proximal lower extremity. There was a 4 to $6 \mathrm{~Hz}$ tremor involving both arms. The tremor was worse on the left than the right and was present mainly at rest. Although rigidity had been reported previously, resistance to passive motion was normal, and he was not hypokinetic. Pinprick was felt less sharply on the right side than on the left, but position sense was intact bilaterally. Vibration was perceived slightly less than normally in the distal portion of the legs. The deep tendon reflexes were symmetrical, but the plantar responses were bilateral extensor.

$A$ radioisotope brain scan and EEG failed to show a focal lesion, and results of a lumbar puncture were normal.

The right hemiparesis completely cleared 24 hours after the patient was admitted to the hospital. Several days later he was alert and underwent additional neuropsychological testing.

\section{SPECIAL TESTS}

Because the patient's signature on hospital forms was reversed in both letter order ("hp"/ ph) and letter orientation (" $q " / p)$ and because examination of signatures obtained during past admissions revealed that this was recent in origin, we examined his reading, writing and orientation for several days, during which time the patient was alert and co-operative.

Writing The patient was asked to use his left hand to write in script the words the examiner presented verbally. Of the 11 words presented, he wrote all from right to left as a mirror image (fig 1A). When each letter of each word was dictated, mirror-writing was evident in all of 8 words totalling 37 letters (fig 1B).

Word order, letter order and letter orientation also were reversed when he wrote sentences. For example, he was asked to write a full sentence telling us his name. He wrote, "My name is Ralph," in mirror-writing (fig 1C).

When using script, it is difficult to write from right to left without reversing the letter orientation. Since we wanted to see what happened to letter orientation independent of word orientation, we asked the patient to print words presented by the examiner. When using his left hand, he printed three words containing a total of ten letters. Although letter orientation was correct, letter order was reversed on two of three words (fig 1D). This was consistent with the finding that when writing multidigit numbers the patient

\begin{tabular}{|c|c|c|}
\hline & Mirror writing & Reverse photography \\
\hline$A$ & no & $\cos$ \\
\hline B & समta & Stove \\
\hline c & Wato of $\sin$ enarlyny & my mone nid s abots \\
\hline$D$ & EEST & TRIJ \\
\hline$E$ & 291 & 1380 \\
\hline
\end{tabular}

Fig 1 Example of patient's mirrorwriting. A, Writing a sentence. $B$, Writing words spelled aloud by examiner. $C$, Writing dictated sentences. $D$, Printing dictated words. E, Printing Arabic numerals. 
reversed digit order but not digit orientation fig 1E). Interestingly, when using his right hand to write a word, he reversed letter orientation of one of three letters, but letter order was correct.

When given alphabet letters to place in order of sequence he did so correctly, but he placed the sequence $A$ to $P$ from left to right and $R$ to $\mathrm{Z}$ from right to left. He was able to spell four of five words correctly by placing the letters in sequence in the correct left to right direction.

In examining the patient's ability to copy, we had him reproduce, with his left hand, words printed for him in the normal left-to-right direction. Of the 13 words presented, a total of 60 letters, he wrote one word in mirror-writing. He printed the remaining words in correct order, but four of the 55 letters were in reverse orientation, a total of nine reversed letters. Seven vertically written words with a total of 32 letters were copied by the patient without any reversals. $\mathrm{He}$ also flawlessly copied five mirror-written words containing 23 letters.

Reading ability Tasks were performed to examine whether the preference for a right-toleft direction of writing also was evident in reading. He correctly followed commands presented graphically, but when asked to read sentences aloud, he read them in reverse word order. This occurred whether or not the sentence made sense when reversed ("Where is it?"/ It is where?; "Name your write."/Write your name.).

An excellent opportunity to view the recovery process of this sinistrad reading of a dextrad language was seen in the tasks requiring reading aloud. Of the 72 words presented over the course of four days, he read ten from right to left. This occurred on both reversible ("saw"/ was) and nonreversible ("nep"/pen) words. However, all ten reversals occurred during the first two days of testing. Thereafter, he read words from left to right.

Reading in reverse direction was not restricted to linguistic stimuli. When reading nine numbers, two to four digits in length, he read seven from right to left ("291"/192) and two from left to right. When given a cue like a dollar sign, the patient read four of eight numbers from left to right.

To investigate whether this man could recognise the orientation of graphic information, we presented letter pairs that included a forward and a backward letter. The letter was named, and the patient was asked to point to the correct one. Eleven of 19 times he chose the letter written backwards. When word pairs were used, three of 11 times he chose the word written backwards. When asked to identify whether a word was written backward or forward, he correctly identified backward written words five of six times and forward written words four of six times.

Orientation We wanted to learn whether this directional confusion was evidenced in the processing of information other than graphic. Therefore, we lcoked at instances in the environment where right/left distinctions were necessary. For example, we asked the patient to indicate on a drawing the direction in which he would run the bases while playing baseball; he drew his course clockwise, opposite to the normal counterclockwise direction, and even labelled the numbers of the bases in mirror image as indicated in fig 2 . When tested again seven days later, he still indicated a clockwise direction for base running.

Directional confusion prevailed when we asked him to identify on a drawing the side of a road he would drive on. He was accurate on this task only two of four times, and when asked to indicate the hot or cold water faucet, he was accurate only one of six times. When tested two and five days later, however, he was accurate $100 \%$ of the time on both tasks.

His directional sense was further investigated by presenting him with pictures and asking questions about them. For example, he was shown a picture of a book and asked which side of the book he would open in order to read it. Of 12 presentations, five were incorrect. One interesting response was in reference to a picture of a telephone. We asked the patient to number each fingerhold on the dial. He began

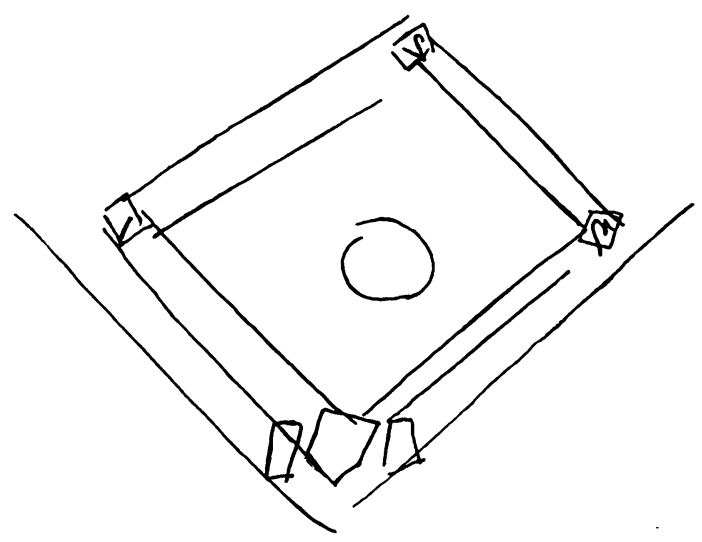

Fig 2 The clockwise direction in which the patient would have run bases in a baseball game. 
with the hole normally marked 0 and labelled it $1 \mathrm{He}$ then proceeded to write the numbers in a clockwise direction; number 2 was on the hole normally labelled 9 , and so forth.

Map usage also was examined. When presented two maps of Florida, one the mirror image of the other, the patient chose the correct one. $\mathrm{He}$ also drew a map of Florida in the correct orientation. When asked to direct the drawing of a map representing a walk around the hospital, he did so accurately. He was presented a map of Florida with numbers indicating the location of cities. Four cities were named by the examiner, and he mislocated two of them. However, we were not certain he could have performed this task before his illness.

This patient's ability to identify right and left parts of his own body or of the examiner's was tested twice. When asked to show his right or left hand, he identified the correct hand in all 20 trials. When given 15 two-part commands, such as put your right hand on your left ear, his performance was also flawless; but when asked to orient to the examiner by pointing to the examiner's right or left hand, the patient was correct on only six of 13 trials (near chance). When tested the next day he had eight of ten trials correct. When given two-part commands, such as "put your right hand on my left hand," he was able to identify his own hand correctly but identified the correct hand of the examiner only nine of 12 times $(75 \%)$ correctly. When tested the next day he identified the examiner's correct hand eight of ten times.

When seen in outpatient clinic three months after the onset of his symptoms, he showed no evidence of mirror-reading or writing.

\section{Discussion}

This patient had the sudden onset of mirrorwriting and reading. His writing was characterised by reversals in both letter order and letter crientation. Reading was equally disturbed. Several theories, including motor and dominance hypotheses, have been proposed to explain these phenomena. We propose also a spatial or orientation hypothesis.

One motor hypothesis of mirror-writing sugrests thit in learning a skilled movement one learns to contract and relax specific muscle groups either simultaneously or sequentially. When using the nonpreferred and untrained hand, if one uses the same motor program as was used by the preferred hand, the movements will have the opposite spatial orientation. ${ }^{1}$ For example, if one makes the letter $\mathrm{C}$ by first flexing and then extending the wrist of the right hand (while simultaneously flexing the first three fingers) and one uses this same movements with the left hand, the $\mathrm{C}$ will be written in a mirror image.

This motor hypothesis is consistent with the finding that some right-handers suffering right hemiplegia may initially write mirror fashion when writing with their left hand. It cannot, however, explain the mirror-writing by this patient because he had always written with his left hand.

A related hypothesis is that mirror movements are the natural movements of the left handi and that they normally are physiologically inhibited. ${ }^{89}$ It is theoretically possible that our subject's left-hemispheric lesion damaged a hypothetical inhibitory centre, thereby allowing mirror-writing to appear.

Another motor hypothesis (which may work in concert with the first hypothesis) suggests that abductive or centrifugal movements are easier to perform and are better controlled than adductive movements; ${ }^{10}$ therefore, when using the left hand, there is a tendency to start from the right and proceed to the left. When we asked our patient to print, his letters were usually oriented in the correct direction; but he continued to print words from right to left, which gives some support to this second motor hypothesis. However, it does not explain why the cerebral infarction induced him to change from adductive to abductive movements. Also, none of these motor hypotheses can explain why our patient had a simultaneous onset of mirrorreading.

Orton $^{11}$ proposed a dominance hypothesis in which visual images in each hemisphere are mirror images of each other. He thought that in normal persons the images lying in the nondominant hemisphere become suppressed. In dyslexic children dominance is less strong and the minor hemisphere is not suppressed, which induces both mirror-writing and reading. Although patients with section of the neocommissure have been studied, ${ }^{12}$ the tendency for them to read in the reverse direction or to reverse letters such as $b$ and $d$ was not reported. To our knowledge, however, each hemisphere of these subjects was not tested to see whether one hemisphere could mirror-read better than the other. In addition, even mirror-writing with the left hand has not been described. ${ }^{12}$

There have been animal studies that partially support Orton's dominance hypothesis, ${ }^{11}$ however. 
To test this hypothesis, one would like to test each hemisphere individually. Because each eye projects to both hemispheres, Noble ${ }^{13}$ transected the optic chiasm in monkeys and induced a state in which each eye projected only to the ipsilateral hemisphere. He next occluded one eye of the animals and trained them to respond to one of a pair of mirror-image objects and then had them perform the same task with the untrained eye; he found they preferred the previously unrewarded mirror image.

Humans, unlike chiasm-sectioned monkeys, usually have visual information presented to both eyes, and each eye projects to both hemispheres. According to Noble, ${ }^{13}$ when monkeys (or men) have primary visual input into both hemispheres (as normally occurs), the primary input to each hemisphere competes with the transcallosal input and the former "overrides" the latter. That humans also have mirror engrams has been demonstrated by Bradshaw, Nettleton and Patterson $^{14}$ and Tankle, ${ }^{15}$ but it is not clear how the primary engrams override the mirror engrams. One explanation is that the engrams formed by primary input override the transcallosal engrams because they are stronger; that is, when the engrams cross the commissure, they may be degraded.

Another possibility is that a right-left spatial system is partly responsible for helping select the correct engram, and a failure of this right-left spatial system may have induced not only mirrorreading but also mirror-writing in our patient.

Ernst Mach $^{16}$ proposed that a symmetrical nervous system could not tell right from left because there would be no reference point. The fact that man can distinguish right from left suggests that an asymmetry exists somewhere in the nervous system. Corballis and Beale ${ }^{17}$ also thought that the ability to discriminate between right and left was based on motor asymmetry or a response asymmetry. Our patient did not have right-left confusion for his own body parts, but did for space. This dissociation between one's own body and space suggests that there must be at least two dissociable factors responsible for discriminating right from left. We possibly use a limb response asymmetry to tell right from left on ourselves. That is, right-handers prefer the right hand because they have motor engrams in their left hemisphere. ${ }^{18} 19$ We may use this preferred hand as a reference for the remaining parts of our body. For directions in space we may use a different strategy. Instead of having a preferred limb as a reference, we may have preferred direction of action or exploration.
When one reads, the direction of a scan remains constant, thus one may learn the directions and positions in space by assessing their temporal relationship. For example, when we read and write English we are taught to scan from left to right. We may also learn that $a d$ is a convex line (c) followed by a straight line (l) and that the letter $b$ is a straight line (1) followed by a concave line. As one reads and scans from left to right and comes upon a convex line followed by a straight line, he will recognise that as a $d$. If, however, one scans from right to left, one will come first to the straight line, then a convex line and will call this $b$ rather than a d. In a similar fashion, letter orders will be reversed. Since mirror image engrams are available, not only will the patient who is scanning from right to left reverse letters and words but may also be capable of reading words written in a mirror fashion. We believe that our patient's left hemisphere lesion induced mirror-reading because it changed his learned direction of exploration.

Similarly, when people learn to write English, they write from their left hemispatial field to their right hemispatial field. In normal people writing on the underside of a table or on the forehead induces mirror-writing. These manoeuvres induce mirror-writing because they induce spatial confusion. When one writes under the table and proceeds from the left to right hemisphere (the ordinary procedure when writing) one neglects to realise that the orientation of the paper is reversed; that is, the portion of the paper on the left side while under the table will be on the right side when the paper is placed in its normal reading position. If one puts a paper under a table and starts in right hemispace and proceeds to left hemispace, the writing will appear more normal.

We believe that in our patient, mirror-writing was induced by spatial confusion. Unlike normal persons who need trick manoeuvres to induce spatial confusion, our patient had spatial confusion induced by his left hemisphere lesion. Although a left-hemispheric lesion appeared to have induced mirror-reading, mirror-writing, and spatial confusion, our patient did have a bilateral extrapyramidal motor system disorder of long standing. He also had a left-sided pallidectomy and thalamotomy. The relative importance of this extrapyramidal disorder in the genesis of mirrorreading, mirror-writing, and spatial confusion remains uncertain.

Mirror-reading and mirror-writing appear to be more common in three populations-the lefthanded ${ }^{20}$ and retarded ${ }^{20}$ and dyslexic children. ${ }^{5}$ 
Left-handers, unlike right-handers, are a heterogeneous population. In their motor skills, they tend to be more ambidexterous than do right-handers, ${ }^{21}$ which suggests that although right-handers have motor engrams stored predominantly in the left hemisphere, left-handers have a great tendency to have bilateral engrams. Unlike right-handers who are strongly left hemisphere-dominant for language, left-handers have a greater tendency to have bilateral language. ${ }^{22}$ Moreover, it appears that the cerebral organisation of left-handers is less asymmetrical than that of right-handers. As mentioned, $\mathrm{Mach}^{16}$ proposed that a symmetrical nervous system could not tell left from right because there would be no reference point. A corollary of Mach's hypothesis might be that less asymmetrical nervous systems have more of a tendency to confuse left and right on themselves and in space. Language processing induces asymmetrical cerebral activation such that in right-handers the left hemisphere is more activated than the right. ${ }^{23}$ Asymmetrical cerebral activation may induce eye deviation; Kinsborne ${ }^{24}$ has shown that when right-handers are processing language, the eyes tend to deviate from left to right. This is the direction in which we are taught to scan when we read. Because some left-handers may have less of an asymmetry of language dominance, when processing language they will have less of an innate tendency to scan in the correct left-to-right direction. It is not surprising, therefore, that a lesion of the left hemisphere, which is responsible for controlling dextrad scanning, would be more likely to reverse learned dextrad scanning patterns.

The incidence of left-handedness is much greater in a population of retarded children than in a population of children of normal intelligence, ${ }^{25}$ which suggests that there are many of these left-handers who are pathological lefthanders (that is, had congenital left-hemispheric lesions). These children should therefore have difficulty learning dextrad scan patterns. In addition, Benton ${ }^{26}$ observed that these children appear to be especially retarded in learning rightleft relations, which could partially explain why these children have a high incidence of mirrorreading and writing.

Lastly, mirror-reading and mirror-writing are also more common in dyslexic children. ${ }^{5}$ These children also often have right-left spatial confusion. ${ }^{27}$ Orton $^{11}$ postulated that the mechanism underlying these disorders was the inability of these children to form a stable dextrad span. Although defective scan may be underlying the children's mirror reversals, it is not clear whether defective scan underlies their reading disability or whether it is just a correlate.

These studies were supported by a grant from the National Institutes of Health, NS-15229. We thank Mrs Alice Cullu for assistance in editing our paper.

\section{References}

1. Critchley M. Mirror writing. London: Kegan Paul, 1928.

2 Fraenkel M. Spiegelschrift und Fehlhandlungen der linken Hand bei Rechtsgelaehmten (Apraxie) Arch Psychiatr 1908; 5:1275-311.

3 Paradowski W, Ginsberg M. Mirror writing and hemiplegia Percept Mot Skills 1971; 32:617-8.

4 Gordon H. Left-handedness and mirror writing. especially among defective children. Brain 1920; 43:313-36.

5 Fischer FW, Liberman IY, Shankweiler D. Reading reversals and developmental dyslexia: a further study. Cortex 1978; 14:496-510.

6 Streifler M, Hofman S. Sinistrad mirror writing and reading after brain concussion in a bisystemic (oriento-occidental) polyglot. Cortex 1976; 12:356-64.

7 Vogt MC. L'écriture considérée au pointe de vue physiologique. Rev Scient 1880; 18:1221-32.

8 Burr CW, Crow CB. Mirror writing and other associated movements occurring without palsy. J Nerv Ment Dis 1913; 40:300-2.

9 Zulch KJ, Muller N. Associated movements in man. Vinken PJ, Bruyn GW, eds. Handbook of Clinical Neurology, Vol. 1. Amsterdam: North Holland Publishing Co, 1949:404-6.

10 Fuller JK. The psychology and physiology of mirror-writing. U Calif Publ Psychol 1916; 2: 199-265. Cited by Carmichael L, Cashman H. J Genet Psychol 1932; 6:296-329.

11 Orton ST. Specific reading disability-strephosymbolia. JAMA 1928; 90:1095-9.

12 Gazzaniga MS. The bisected brain. New York: Appleton Century Crofts, 1970:117-20.

13 Noble J. Paradoxical interocular transfer of mirror-image discriminations in the optic chiasm sectioned monkey. Brain Res 1968; 10:127-51.

14 Bradshaw JL, Nettleton NC, Patterson K. Identification of mirror-reversed and nonreversed facial profiles in same and opposite visual fields. J Exp Psychol 1973; 99:42-8.

15 Tankle RS. Mirror image confusion in adults and children. Presented at the International Neuropsychological Society. New York, Februarv. 1979.

16 Mach E. Popular scientific lectures. Chicago Open Court Publishing House, 1894.

17 Corballis MC, Beale IL. Psychol Rev 1970; 77: 451-64.

18 Liepmann H. Du linke Hemisphäre und das Handeln. Drei Aufsatre dem Aufsatze dem A praxiegebeit. Berlin: Karger, 1908; 17-50. 
19 Liepmann H, Maas O. Fall von linksseitiger Agraphie und Apraxie bei rechtsseitiger Lähmung. J Psychol Neurol 1907; 10:214-27.

20 Carmichael L, Cashman H. A study of mirror writing in relation to handedness and perceptual and motor habits. J Genet Psychol 1932; 6:296329.

21 Benton AL. Clinical symptomatology in right and left hemisphere lesions. In: Mountcastle VB, ed. Interhemispheric relations and cerebral dominance. Baltimore: Johns Hopkins Press, 1962: 253-63.

22 Satz P. A test of some models of hemispheric speech organisation in the left- and right-handed. Science 1979; 203:1131-33.
23 Galin D, Ellis R. Asymmetry in evoked potentials as an index of lateralised cognitive processes: relation to EEG alpha asymmetry. Neuropsychologia 1975; 13:45-50.

24 Kinsbourne $M$. Eye and head turning indicates cerebral lateralisation. Science 1972; 176:539-41.

25 Hécaen $\mathrm{H}$, de Ajuriaguerra J. Left Handedness: manual superiority and cerebral dominance. New York: Grune \& Stratton, 1964.

26 Benton AL. Right-left discrimination and finger localisation: development and pathology. New York: Hoeber-Harper, 1959.

27 Croxen ME, Lytton $H$. Reading disability and difficulties in finger localisation and right-left discrimination. Dev Psychol 1971; 5:256-62. 